\title{
Conciencia ambiental desde la educación: Estado del Arte
}

Environmental awareness from education: State of the Art

\section{María Elena Rubina Ticlla}

Licenciada en Educación primaria, Maestra en Psicología educativa, Técnico en administración de empresas Docente, IE Villa Emilia de MP, mrubinat12@ucvvirtual.edu.pe, marilenarubi40@gmail.com, ORCID_https://orcid.org/0000-0002-2969-4484. https://scholar.google.com/citations?user=mCALV84AAAAJ\&hl=es

\section{Jesús Emilio Agustín Padilla Caballero}

Docente, Maestro en docencia y gestión educativa, Segunda especialidad en Evaluación de la calidad de los aprendizajes, Doctor en administración de la educación, PhD. en Ética y Responsabilidad Social y derecho Humanos, Docente Investigador y Temático, Universidad César Vallejo, Correo electrónico institucional: jpadillac@ucv.edu.pe, Correo electrónico personal: claimvenst2@yahoo.com, ORCID https://orcid.org/0000-0002-9756-8772, Google Académico: https://scholar.google.com/citations?user=8AehlgwAAAAJ\&hl=es

\section{María Cristina Gutiérrez Cárdenas}

Licenciada en educación secundaria, Maestra en Docencia y Gestión Educativa, subdirectora de nivel secundario IE 2088 República Federal de Alemania de SMP, mcgutierrezc@ucv.edu.pe, maricrisscardenas@gmail.com, https://orcid.org/0000-00026803-3576, https://scholar.google.es/citations?user=W6c9FSoAAAAJ\&hl=es

Carmen Espinal Farfán

Licenciada de Educación secundaria, Maestra en Docencia y Gestión Educativa. Docente de Comunicación en la IE "Gran Mariscal Toribio de Luzuriaga", Los Olivos. Cespinalf@edu.ucv, espinal.gmtl@gmail.com , ORCID http://orcid.org/0000-00002-35283480 , Google académico https://scholar.google.es/scholar?hl=es\&as_sdt=0\%2C5\&q=carmen+espinal+farfan\&btnG=

\section{Revista Iberoamericana de la Educación Vol - Especial 12021 \\ e-ISSN: 2737-632x}

Resumen: La conciencia ambiental es un constructo complejo de conocimientos, abordados por diferentes ciencias, como: las sociales, las económicas, las políticas, etc., pero aún no se entiende ni comprende en su correcta dimensión. El objetivo de este estado del arte es describir hasta qué punto se ha investigado sobre la conciencia ambiental en la educación, específicamente en estudiantes de educación primaria y secundaria en 
diferentes países. El método empleado es de acopio, análisis e interpretación de toda la literatura científica encontrada durante el estudio. Es así que, se analizaron 30 artículos en diferentes bases de datos indexadas, sobre componentes, estrategias, programas y niveles de la conciencia ambiental. Así mismo, se consideró la temática, método, muestra, instrumentos y resultados. Los datos fueron tratados a través de la estadística descriptiva y un software cualitativo. Los resultados obtenidos sobre la conciencia ambiental de los estudiantes de educación primaria y secundaria presentaron categorías como: (1) Conservación climática, (2) Conservación de la biodiversidad, (3) Protección de la biodiversidad, (4) Protección de la tierra, (5) Conservación hídrica, y (6) Nivel alto de conciencia ambiental.

Palabras clave: conciencia ambiental, componentes ambientales, estrategias de conservación, programas ambientales, protección ambiental.

Abstract: Environmental awareness is a complex construct of knowledge, addressed by different sciences, such as: social, economic, political, etc., but it is not yet understood or understood in its correct dimension. The objective of this state of the art is to describe the extent to which environmental awareness in education has been investigated, specifically in primary and secondary school students in different countries. The method used is the collection, analysis and interpretation of all the scientific literature found during the study. Thus, 30 articles were analyzed in different indexed databases, on components, strategies, programs and levels of environmental awareness. Likewise, the theme, method, sample, instruments and results were considered. The data were treated through descriptive statistics and qualitative software. The results obtained on the environmental awareness of primary and secondary education students presented categories such as: (1) Climate conservation, (2) Biodiversity conservation, (3) Biodiversity 
protection, (4) Land protection, (5) Water conservation, and (6) High level of environmental awareness.

Keywords: environmental awareness, environmental components, conservation strategies, environmental programs, environmental Protection

\section{Introducción}

La conciencia ambiental es la actitud, la acción y el saber sobre los efectos de la actividad humana en favor o en contra del equilibrio medioambiental. Durante la época de pandemia se ha identificado un incremento de la conciencia ambiental en los estudiantes y comunidad debido a la presencia de ríos cristalinos, mares con presencia de biodiversidad, ciudades invadidas por especies salvajes y silvestres, atmósfera limpia y descenso en los índices de contaminación de suelos, aguas y aire (Cerillo, 2020). En el mismo sentido se pronunció el Banco Mundial al sostener que la conservación atmosférica es elemental para evitar la propagación de enfermedades (Narain, 2020). Sin embargo, hubo incremento del consumo de material plástico en ambientes domésticos y hospitalarios (Dalhey, 2020).

En el Perú, el aislamiento social obligatorio, afectó siembras, ganado, granjas, programas de reforestación (Dourojeanni, 2020). En consecuencia, la descontaminación, la presencia de fauna en ciudades, la pureza atmosférica llevó a la reflexión y comprensión de la protección del medio ambiente, influyendo en el nivel de conciencia ambiental de alumnos y comunidad.

El desarrollo de la conciencia ambiental requiere de estrategias en todos los niveles para hacer frente a la problemática generada por la contaminación. La mejor estrategia fue catalogar la adversidad como una oportunidad de aprendizaje y racionar energía, agua, consumir productos frescos y enseñar a los escolares a valorar la naturaleza (Educa 2021). A nivel mundial, la ONU - Programa para el Medio Ambiente (PNUMA) declararon dos estrategias: 
(1) brindar todo el apoyo a la Agenda 2030 con los Objetivos de Desarrollo Sostenible considerando que es la mejor oportunidad para lograr un futuro mejor, y (2) hacer un replanteamiento de la relación del hombre con la naturaleza y dar inició a la construcción de un mundo mejor (ONU-PNUMA, 2020).

Por su parte el Perú a través del Ministro de Educación exhortó a la ejecución del Plan Nacional de Educación Ambiental 2016-2021 PLANEA (Minedu, 2020) y el Ministerio del ambiente consideró seis estrategias para la preservación medioambiental, referidas a la reducción de los problemas de contaminación; promoción de inversiones que aseguren la calidad ambiental; protección y puesta en valor de la biodiversidad; construcción de un país climáticamente responsable; desarrollo de instrumentos y herramientas para valorar la riqueza ambiental; valoración del bosque en pie y generación de mecanismos para la prevención de la deforestación y conservación de los ecosistemas (Ministerio del Ambiente, 2021). Por lo tanto, las estrategias deben generarse en todos los niveles para preservar el medio ambiente.

Para generar conciencia ambiental se requieren de programas de gran envergadura. En este sentido, se cuenta con el programa: Un mandamiento verde: "no ocasionarás daños" ejecutado por Unión Europea, en el que se realizan consultas públicas; identificación de las repercusiones medioambientales, sociales y económicas, dictan medidas para la mejora de la legislación y las herramientas de apoyo; cubren las cuestiones relacionadas con la sostenibilidad y la innovación; así mismo, el 2020 se inició la ejecución del marco del Pacto Verde como parte del VIII Programa de Acción en materia de medio Ambiente (García, Jordano, Lozano y Nogueira, 2020).

El Ministerio de Agricultura retomó el Programa GLOBE, relacionado a la educación ambiental con enfoque científico, cuyo objetivo es desarrollar el 
interés por las ciencias y la investigación ambiental, para apropiarse de su entorno y contribuir a la solución de problemas ambientales; en las escuelas se promueve la participación en la solución de problemas asociados a fenómenos climáticos, al manejo de los recursos hídricos y a la gestión de la biodiversidad, para el 2020, 50 escuelas participaban en el Programa (Ministerio del Ambiente, 2021a). Por lo tanto, tanto las organizaciones mundiales como las nacionales deben promover la ejecución de programas de desarrollo de la conciencia ambiental y deben asignarle los recursos necesarios.

Los niveles de conciencia ambiental, se refieren al grado de conocimiento teórico práctico que tiene una persona respecto a la protección y contaminación del medio ambiente. Se encuentra que la Organización Panamericana de la Salud (OPS) precisa que, la consecuencia del bajo nivel de conciencia ambiental en la comunidad, ha originado que 83 millones de personas vivan sin servicios de saneamiento mejorado; vivan expuestas a riesgos químicos, por la exposición a plaguicidas tóxicos, plomo y mercurio los que afectan el desarrollo neurológico y producen enfermedades respiratorias, cardiovasculares, traumatismos y defunciones prematuras las que se agravarán con los nuevos peligros de desechos electrónicos, nanopartículas, micro plásticos, productos químicos que alteran el sistema endócrino y escasez de agua (OPS, 2021). En una investigación desarrollada en el Departamento de San Martín, se determinó que en el nivel de ambientalización curricular y en la toma de conciencia ambiental, los estudiantes se ubican en el nivel medio de conciencia ambiental (Santacruz, 2018). Como se ve, los datos muestran que hay un bajo nivel de conciencia ambiental en la región y en el país. 
Desde el punto de vista teórico la conciencia ambiental es concebida como una actitud general hacia el objeto de protección que es el medio ambiente (Bamberg, 2003); que contiene un componente activo y otro cognitivo, asociados a la intencionalidad de realización de conductas proambientales, al implicarse en asuntos del medio ambiente (Ohtomo e Hirose, 2007); los componentes intelectual y afectivo requieren el conocimiento de su problemática (Ranniko, 1996); en ese sentido, la conciencia ambiental son los saberes y la sensibilización con relación a los problemas medioambientales (Ruiz, 2006).

La conciencia ambiental tiene dos ideas fuertemente arraigadas, sea como actitud o como valoración de la visión del mundo. En el primer caso, la idea de actitud parte de la propuesta de Maloney y Ward (1973) quienes desarrollaron la primera escala para la medición de la actitud ecológica y consideraron los componentes: afectivo, compromiso verbal, compromiso real y conocimiento de la problemática. En el segundo caso, se trata de una representación, de una renovada forma de pensamiento de las relaciones de la naturaleza con el ser humano a la que denominaron el paradigma ecológico, propuesta inspirada en los trabajos de Van Liere y Dunlap (1981) y presentada por Mathies y Blöbaum (2007).

El abordaje de la conciencia ambiental es multidimensional y en ese sentido se ha desarrollado desde diferentes miradas siendo las más frecuentes los estudios sociales, económicos, políticos, psicológicos, tecnológicos los cuales se encuentran más vinculados a la problemática medioambiental (Kilbourne y Polonbsky, 2005). En el análisis de los problemas se ha priorizado la afectación del hombre como consecuencia de las alteraciones medioambientales y no en sentido opuesto, es decir, cuanto afecta la acción del hombre a la naturaleza (Schultz, 2002; y Dutcher, Finley, Luloff y Johnson, 2007). 
La presente investigación se orienta por el objetivo de describir la conciencia ambiental en los estudiantes de educación básica regular.

\section{Materiales y métodos}

El 26,7 \% de las investigaciones consultadas corresponden en forma predominante a niveles de conciencia ambiental (CA); una cifra similar a ejecución de programas de CA; 23,3\% se refieren a estrategias para su implementación; y otro 23,3 \% a los componentes de la CA. El tipo de muestre que emplearon los investigadores en el 76,7 \% fue no probabilístico y el 23,3 \% fue probabilístico. El 83,3\% de las investigaciones trabajaron con muestras de estudiantes; el 13,3 \% se refieren a trabajos de revisión y el 3,3 $\%$ tomaron a estudiantes y docentes en la muestra. En cuanto al método de investigación empleado en los trabajos consultados, el 43,3\% corresponden a métodos descriptivos; $23,3 \%$ a cualitativos de tipo investigación acción; $16,7 \%$ emplearon métodos mixtos; $13,3 \%$ fueron trabajos experimentales; $y$ 3,3\% cualitativos de tipo hermenéutico. En cuanto al tipo de instrumento que se empleó, el 36,7 \% uso cuestionario; el $20 \%$ lista de cotejo; 16,7 \% emplearon dos o más instrumentos; $13,3 \%$ empleó entrevista; $10 \%$ tabla de categorización; y 3,3\% escala.

Esta investigación es de revisión de literatura científica, para ello se seleccionaron 30 artículos de investigación de bases de datos indexadas: Scopus, EBSCO, ProQuest y Redalyc, agrupadas según la temática de conciencia ambiental. Los criterios de búsqueda que correspondan al 20192020, sin embargo, se incluyeron cuatro investigaciones de fecha anterior al no encontrarse material reciente sobre dichas dimensiones. La información acopiada ha sido sometida a análisis a través de un software y luego a un 
análisis de contenido, se ha mantenido la coherencia con la temática y está alineada al objetivo de la investigación.

\section{Resultados}

Análisis categorial de la Conciencia Ambiental (CA)

Figura 1. Componentes de la conciencia ambiental

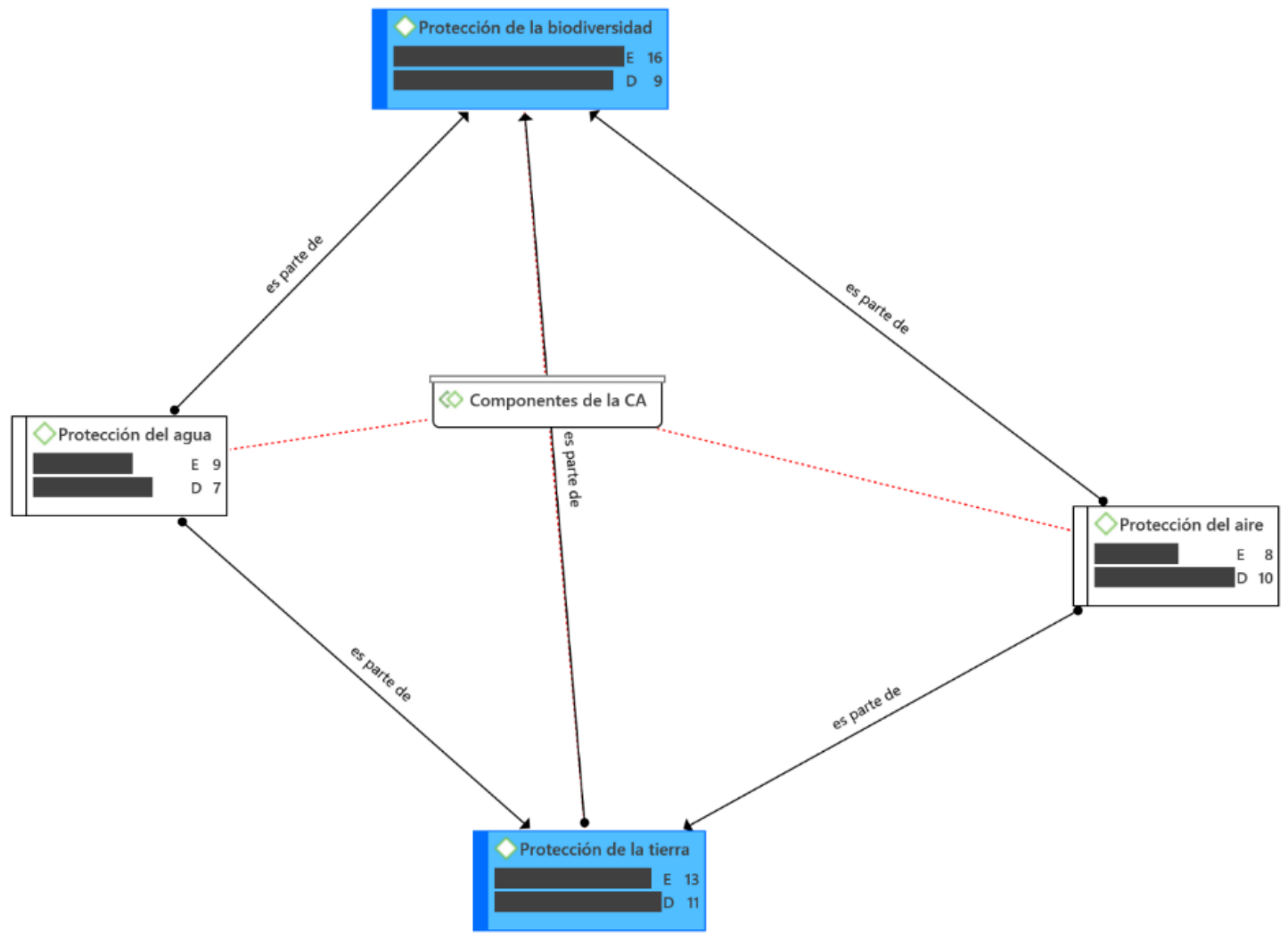

Según los trabajos consultados, reportan un mayor índice la protección de la biodiversidad y la protección de la tierra, seguidos de la protección del aire y la protección del agua.

Figura 2. Estrategias de la conciencia ambiental 


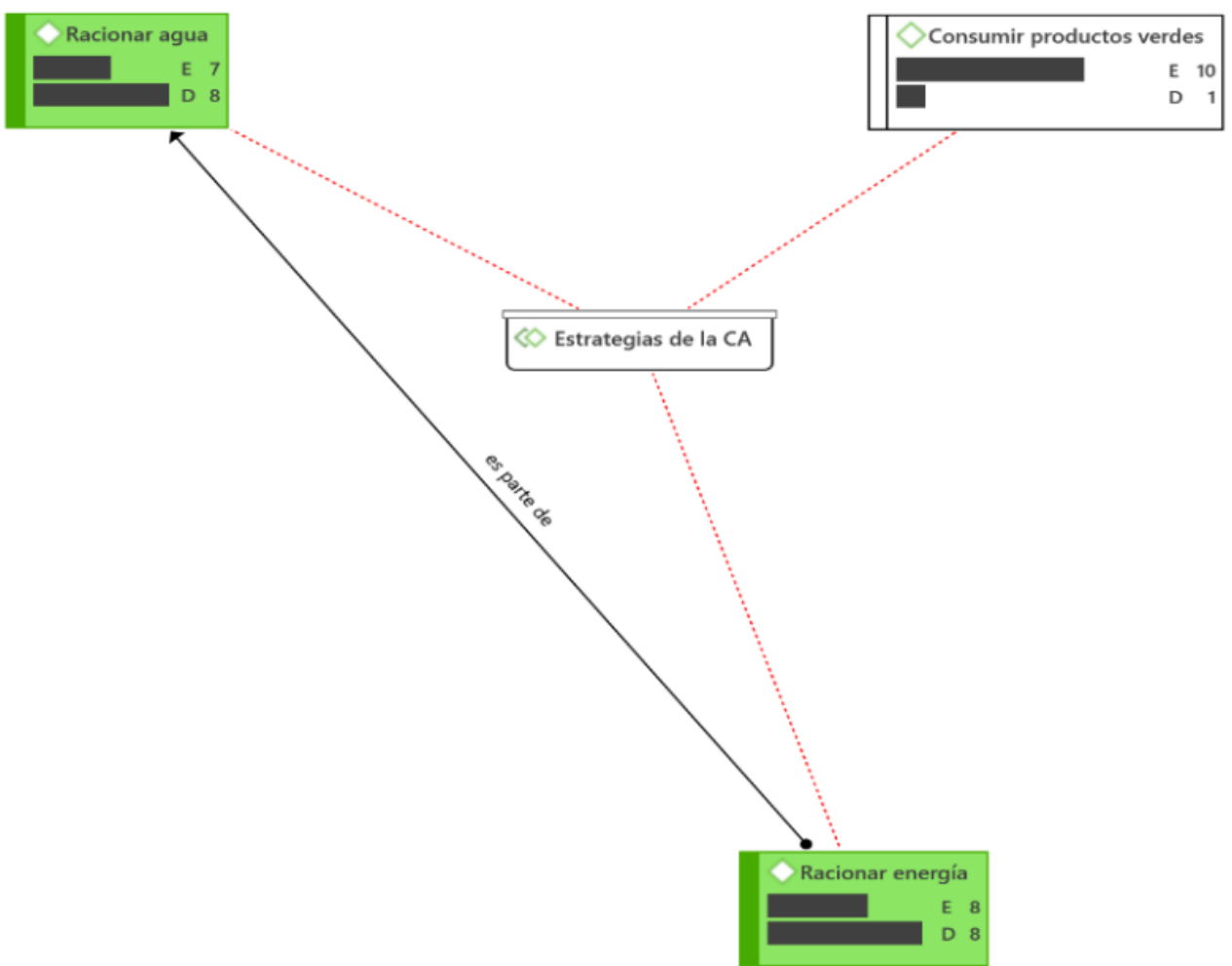

El mayor índice se concentra en el racionamiento del agua, seguido de racionamiento de energía y el consumo de productos verdes.

Figura 3. Programas de desarrollo de la conciencia ambiental

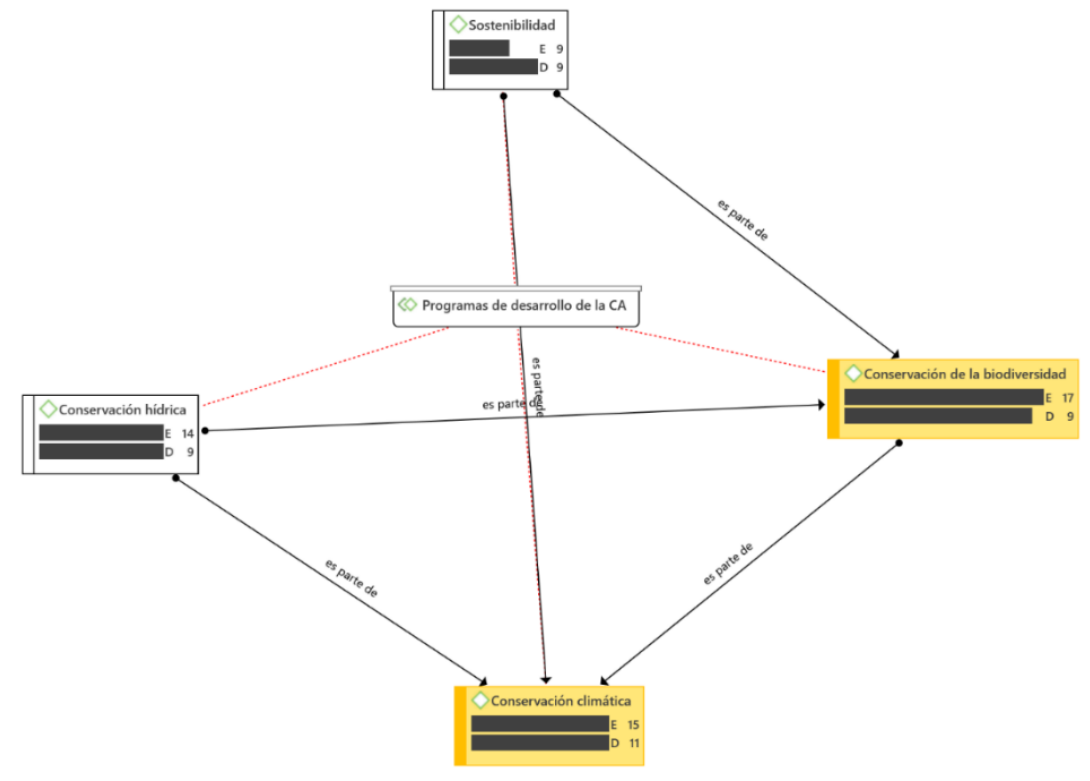


En los programas de conciencia ambiental la conservación de la biodiversidad y la conservación climática, seguidos de la conservación hídrica y la sostenibilidad

Figura 4. Niveles de conciencia ambiental

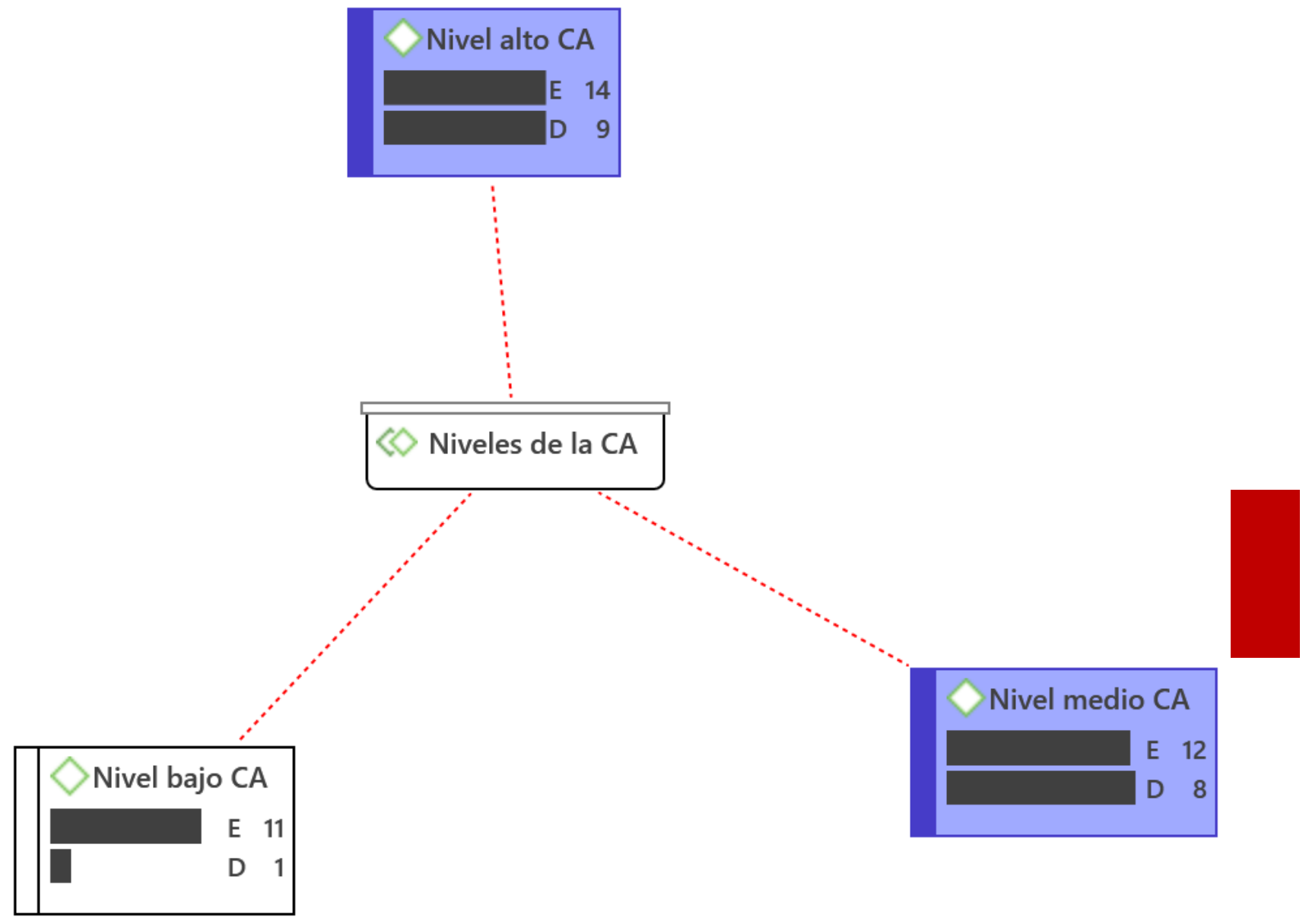

Destaca un nivel de conciencia ambiental alto, seguido del nivel medio y en último puesto se ubica el nivel bajo.

Figura 5. Triangulación de autores sobre conciencia ambiental 


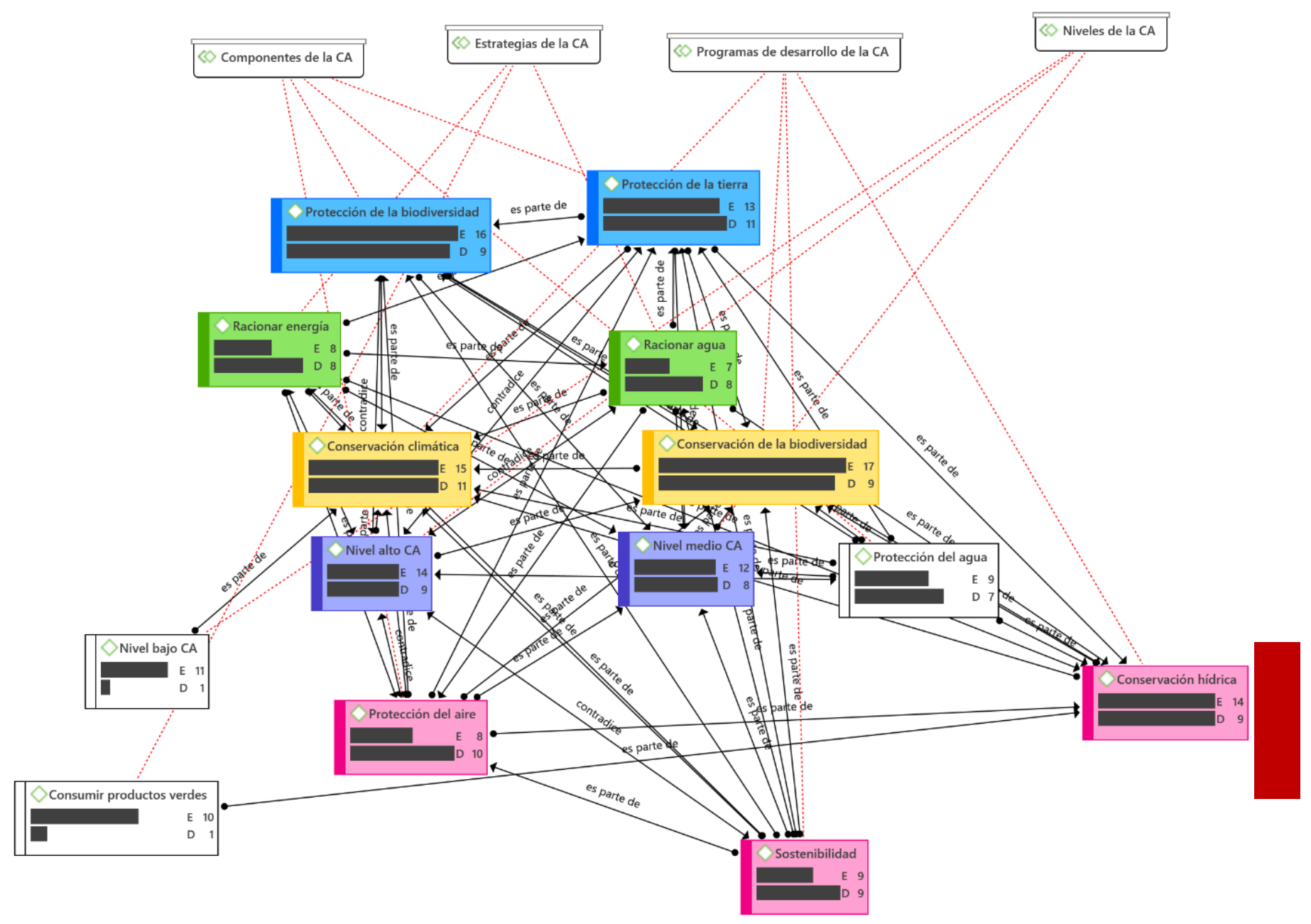

Existen coincidencias en los autores consultados en: (1) Conservación climática, (2) Conservación de la biodiversidad, (3) Protección de la biodiversidad, (4) Protección de la tierra, (5) Conservación hídrica, y (6) Nivel alto de conciencia ambiental.

\section{Discusión}

Se encontró que sobre los componentes de la conciencia ambiental, los trabajos consultados que reportan un mayor índice son: la protección de la 
biodiversidad, lo que equivale a que los estudiantes consideran a la flora y la fauna como prioridades, luego aparece la protección de la tierra asociada a minería, erosión, tala de bosques, depredación; la protección del aire está referida a la contaminación atmosférica, capa de ozono, contaminación sonora; y la protección del agua tiene relación a los relaves, basura que se arroja a los ríos e inadecuado tratamiento de las aguas residuales de alcantarillado. Estos resultados con coincidentes con los hallazgos de Chuliá, (1995); y Miranda (2017) quienes encontraron que la actitud de la persona con el medio ambiente en la búsqueda de una protección recíproca; es una relación entre sociedad - medio ambiente, resultante de procesos cognitivos, afectivos, conativos y activos. En el mismo sentido Hannigan (2006) sostuvo que la CA aparece en la agenda cuando se presentan problemas como consecuencia de reclamaciones y hallazgos científicos, como la lluvia ácida, la pérdida de biodiversidad, el calentamiento global, el agujero en la capa de ozono, la desertificación y el envenenamiento por dioxinas, dentro de otras. Corraliza et al. (2000) precisaron que lo que más mueve a la promoción de la conciencia ambiental son los problemas de la degradación y disminución de los recursos naturales que son causados por el efecto de la contaminación. Y Santacruz (2018) indicó que la conservación del medio ambiente es un fenómeno complejo que compromete diferentes disciplinas y posturas y a nivel individual se convierte en un estilo de vida.

Así mismo, respecto a los componentes de la conciencia ambiental, Amran et. al (2019) señalaron que los alumnos de educación media en actitudes y conciencia ambiental en las dimensiones de pensamiento crítico, pensamiento creativo, comunicación y colaboración, se encontró que en todas ellas se ubicaron en el nivel bajo. Koyunlu Ünlü (2020) precisó que se evaluó los componentes conductual y actitudinal, hacia la limpieza del ambiente de trabajo y concluyó que los componentes conductual y actitudinal estaban 
presentes en el discurso narrativo, pero eran incoherentes con el estilo de vida que llevaban los educandos. Çelik (2020) encontró que los estudiantes en sus aprendizajes eran predominantemente teóricos por lo que era necesario ofrecer experiencias para fortalecer las estructuras cognitivas. Nunes et al. (2020) hallaron que los educandos no conocían (cognitivo) los perjuicios que causaba el desechar las bolsas de plástico y la carencia de un componente cognitivo repercutía en la actitud frente al problema, con lo cual agravaban el problema de la contaminación

Del mismo modo, Punzalan et al. (2019) encontró que los estudiantes de regiones urbanas tienen un mayor nivel de conciencia ambiental como consecuencia del ambiente en el que viven donde hay acumulación de basura, contaminación sonora, polución. Castro et al. (2016) encontraron que las percepciones de los estudiantes de zonas marginales resultaban desfavorables a la conservación del medio ambiente, por lo que se recomendó su promoción a través de actividades lúdicas. Santos y Passarella (2013) hallaron que la sola capacitación resultó insuficiente para movilizar la conciencia ya que se requería de recursos de diferente índole que permitan vivir con dignidad, justicia social, tolerancia y evitación de exclusión social. Y Süklüm (2019) halló que, si bien los educandos tenían conocimientos, carecían en absoluto de conciencia, no estaban formados para promover la conciencia ecológica.

En lo relacionado a las estrategias de conservación medio ambiental, se reporta que el mayor índice de los hallazgos encontrados en los trabajos de investigación se concentran en el racionamiento del agua debido a la sequía que se produce en algunas regiones, a la falta de acceso a los servicios de agua en algunas partes de la ciudad y a su desperdicio a través de los ríos; seguido de racionamiento de energía la acula no se administra de forma equitativa, algunos acceden a volúmenes elevados de energía, mientras otros carecen de 
ella, de manera indirecta hay referencias al uso de energía renovables; y el consumo de productos verdes que aparece con un buen nivel de enraizamiento pero con baja densidad, por lo que se entiende que los jóvenes están cambiando sus percepciones respecto a los hábitos de alimentación. Estos hallazgos tienen semejanzas con las investigaciones de Leite y Lopes (2020) quienes encontraron que se valoró la importancia del suelo como sustrato para la vegetación, la vida de animales y la vida de los seres humanos. Araya et al. (2019) quienes hallaron una situación incierta y negativa respecto al futuro, la toma de conciencia de los estudiantes, generó percepciones de la responsabilidad de los gobiernos de asignar recursos para la implementación de proyectos en las que se comprometan todos los actores sociales para la conservación de los ecosistemas. Martin et a. (2019) hallaron que cuando se combinan espacios de aula y aire libre y se incorpora en forma progresiva diversas formas de enseñar a través de los escenarios virtuales; la forma de impartir clases en las zonas rurales permanece igual, por lo que es necesario contextualizar el desarrollo de las clases para generar conciencia ambiental del estudiante con su ecosistema situado

En el mismo sentido sobre las estrategias de conciencia ambiental, Ahn (2020) concluyó que la conciencia ambiental se promueve desde diferentes espacios y a través de diversas estrategias educativas y que el material audiovisual a través de documentales y cortometrajes resulta altamente persuasivo de la responsabilidad del ser humano para la protección del medio ambiente.

Andrade y Gonzales (2019) encontraron que en efecto los conocimientos teóricos sobre basura, energía, uso de espacios y obligación moral, no se relacionaban de manera objetiva con su comportamiento sobre reciclaje de basura, ahorro de energía y preservación de los espacios, resultando en desventaja los estudiantes de zonas rurales quienes desconocen las técnicas 
para la eliminación de residuos y para las acciones de reciclaje. Tonello y Valladares (2015) encontraron que en estudiantes con quienes se investigó el uso racional de la energía para la iluminación quienes entendían la responsabilidad humana en este aspecto en función al grado de conocimiento que tenían sobre las acciones ambientales que se recomendaban para la energía de iluminación se logró un cambio de actitud. Y Martínez et al. (2020) precisaron que el tiempo de ocio de los adolescentes cuando lo disfrutan en contacto con la naturaleza, favorece una conexión emocional con esta y promueve el desarrollo de pensamiento proambiental desde la reflexión, por lo que, resulta en una buena estrategia favorecer el uso del tiempo de ocio en entornos naturales para una restitución de la armonía entre las personas y su ambiente.

En lo que concierne a los programas de desarrollo de la conciencia ambiental se encontró que aparece como prioridad la conservación de la biodiversidad, en este caso se hace alusión a las especies que deben ser protegidas para evitar su migración o extinción; la conservación climática relacionada a la combustión de hidrocarburos y emisión de gases tóxicos, seguidos de la conservación hídrica la cual requiere la reforestación y la sostenibilidad para que los programas no tengan una existencia efímera, toda vez que se requiere que estén institucionalizados. Estos resultados son semejantes a los de Izquierdo et al. (2010) quienes encontraron que la simulación en escenarios reales en los que se incluyen aspectos formales de la currícula y narrativa de los pobladores, permite el desarrollo de una elevada conciencia ambiental y la predisposición para actuar cuando esta se presente. Robina y Medina (2019) reportaron que la aplicación de programas en los que los estudiantes conozcan y valoren las condiciones de riesgo de las comunidades, son efectivas en la generación de conciencia ambiental y prevención de riesgos. 
Amérigo et al. (2017) precisaron que la conciencia ambiental es multidimensional en la medida que hace una evaluación de las relaciones de las personas con su entorno, incluye por lo menos estas cuatro dimensiones; (1) apatía, (2) antropocentrismo, (3) conectividad, y (4) afinidad emocional. Santos et al. (2014) hallaron que las actitudes e intenciones de uso de bolsas de plástico no reciclables en estudiantes que tenían conocimiento que las bolsas no eran reciclables, le daban usos adicionales, por lo que se demostró que no existía relación entre las actitudes frente a las bolsas no reciclables y el comportamiento de las personas.

Del mismo modo, en lo relacionado a los programas de desarrollo de la conciencia ambiental, Ghanem y Garay (2017) encontraron que a menor edad había una mayor motivación e interés y empleaban su creatividad. Espejel et al. (2017) reportó que las actividades vivenciales inducen a la reflexión, logran el compromiso de los educandos quienes desarrollan acciones de prevención en su círculo de pertenencia y en su comunidad. Widiyawati (2020) encontró que tanto las zonas industriales como las zonas ganaderas los focos de mayor peligro para el cambio climático son los residuos químicos. Y Kurniawan y Sari (2019) en un programa desarrollado para promover la regeneración ambiental desde la escuela a través del liderazgo escolar con conocimiento de riesgos y con altos niveles de conciencia ambiental, encontraron que no hay una educación sostenida sobre medio ambiente, los temas se abordan de manera aislada y no existen docentes comprometidos con la promoción sostenida de actividades destinadas a la aplicación de regeneración después de ocurridos los desastres, por lo que es necesaria la formación de cuadros.

En referencia a los niveles de conciencia ambiental, se encontró que los estudiantes reportan un nivel de conciencia ambiental alto, esto podría 
explicarse por la importancia que ha suscitado el tema en los últimos años, si bien no hay acciones puntuales que desarrollan los estudiantes, ya tienen conciencia que el medio ambiente está en peligro; luego se ubican los estudiantes que tienen un nivel medio de conciencia ambiental, y según el reporte de las investigaciones consultadas, en último puesto se ubica el nivel bajo. Estos resultados son congruentes con los hallazgos de Laso et al. (2019) concluyeron que a nivel experimental, un programa de metacognición favorece el desarrollo de la conciencia ambiental reportando nivel alto de conciencia ambiental. Aliman et al. (2019) encontraron que luego de experimento con estudiantes de básica a quienes se les aplicó un programa específico para afrontar problemas se demostró que los programas experimentales para generar conciencia ambiental, a nivel experimental, resultaron efectivos logrando un nivel de conciencia ambiental alto. Danielraja (2019) indicó que en una investigación en la que se aplicó el mismo programa a estudiantes de ciencia y de artes y los resultados mostraron que los que pertenecían al área de ciencias alcanzaron puntuaciones elevadas, en tanto los estudiantes de arte alcanzaron puntuaciones pobres. Rogayan y Nebrida (2019) encontraron que no existía tal relación entre conceptos y conciencia ambiental, con excepción de los estudiantes que mostraban altas calificaciones en las áreas de ciencias, en cuyo caso el dominio de constructos conceptuales estaban relacionados con la conciencia sobre la problemática del medio ambiente. Yeşilyurt et al. (2020) encontraron que mientras más se involucran con la conciencia ambiental tienen una mejor presentación, la estética es más refinada y la expresión visual contiene su compromiso con el medio ambiente.

En los niveles de conciencia ambiental, se encontraron resultados similares en el trabajo de Amran et al. (2019) en el programa de educación para un desarrollo sostenible en cuatro dimensiones: (1) pensamiento crítico, (2) 
colaboración, (3) comunicación, y (4) pensamiento creativo, sin embargo, obtuvieron puntuaciones bajas. Gomera et al. (2013) encontraron que las dimensiones que eran mejores predictoras de la conciencia ambiental fueron: (1) afectiva, (2) cognitiva, (3) actitudinal o conductual. E, Yilmaz (2019) quien empleó dos escalas de conciencia ambiental en un estudio comparativo entre colegios de gestión pública y los de gestión privada, encontró que, tanto en la escala de conciencia ambiental como en la escala de habilidades del proceso científico básico, los resultados altos estaban a favor de las escuelas privadas, situación que podía comprobarse desde la observación por el desempeño de los estudiantes.

En la triangulación de autores sobre conciencia ambiental, se encontró que se validan las siguientes subcategorías en el orden que aparecen: (1) Conservación climática, (2) Conservación de la biodiversidad, (3) Protección de la biodiversidad, (4) Protección de la tierra, (5) Conservación hídrica, y (6) Nivel alto de conciencia ambiental. Las demás subcategorías son calificadas como ordinarias debido a que su índice de emergencia está en el promedio o por debajo de este.

\section{Conclusiones}

En la triangulación de autores sobre conciencia ambiental, se encontró que se validan las siguientes subcategorías en el orden que aparecen: (1) Conservación climática, (2) Conservación de la biodiversidad, (3) Protección de la biodiversidad, (4) Protección de la tierra, (5) Conservación hídrica, y (6) Nivel alto de conciencia ambiental. Las demás subcategorías son calificadas como ordinarias debido a que su índice de emergencia está en el promedio o por debajo de este.

Se encontró que sobre los componentes de la conciencia ambiental que reportan un mayor índice son: la protección de la biodiversidad, lo que 
equivale a que los estudiantes consideran a la flora y la fauna como prioridades, luego aparece la protección de la tierra asociada a minería, erosión, tala de bosques, depredación; la protección del aire está referida a la contaminación atmosférica, capa de ozono, contaminación sonora; y la protección del agua tiene relación a los relaves, basura que se arroja a los ríos e inadecuado tratamiento de las aguas residuales de alcantarillado.

En lo relacionado a las estrategias de conservación medio ambiental, se reporta que el mayor índice de los hallazgos encontrados en los trabajos de investigación se concentran en el racionamiento del agua debido a la sequía que se produce en algunas regiones, a la falta de acceso a los servicios de agua en algunas partes de la ciudad y a su desperdicio a través de los ríos; seguido de racionamiento de energía la acula no se administra de forma equitativa, algunos acceden a volúmenes elevados de energía, mientras otros carecen de ella, de manera indirecta hay referencias al uso de energía renovables; y el consumo de productos verdes que aparece con un buen nivel de enraizamiento pero con baja densidad, por lo que se entiende que los jóvenes están cambiando sus percepciones respecto a los hábitos de alimentación.

En lo que concierne a los programas de desarrollo de la conciencia ambiental se encontró que aparece como prioridad la conservación de la biodiversidad, en este caso se hace alusión a las especies que deben ser protegidas para evitar su migración o extinción; la conservación climática relacionada a la combustión de hidrocarburos y emisión de gases tóxicos, seguidos de la conservación hídrica la cual requiere la reforestación y la sostenibilidad para que los programas no tengan una existencia efímera, toda vez que se requiere que estén institucionalizados.

En referencia a los niveles de conciencia ambiental, se encontró que los estudiantes reportan un nivel de conciencia ambiental alto, esto podría explicarse por la importancia que ha suscitado el tema en los últimos años, si 
bien no hay acciones puntuales que desarrollan los estudiantes, ya tienen conciencia que el medio ambiente está en peligro; luego se ubican los estudiantes que tienen un nivel medio de conciencia ambiental, y según el reporte de las investigaciones consultadas, en último puesto se ubica el nivel bajo.

\section{Referencias}

Ahn, C. (2020). Considering the Role of Documentary Media in Environmental Education. Journal of the Canadian Association for Curriculum Studies, 17(2),

$67-79$. https://jcacs.journals.yorku.ca/index.php/jcacs/article/view/40435

Aliman, M., Budijanto, Sumarmi, \& Astina, I. (2019). Improving Environmental Awareness of High School Students in Malang City through Earthcomm Learning in the Geography Class. International Journal of Instruction, 12(4), 79-94. https://files.eric.ed.gov/fulltext/EJ1230052.

Amérigo, M., García, J., \& Côrtes, P. (2017). Análise Das Atitudes E Comportamentos Pró-Ambientais: Um Estudo Exploratório Com Uma Amostra De Estudantes Universitários Brasileiros. Ambiente \& Sociedade, 20(3), 1-19. https://doi.org/10.1590/18094422ASOC300R1V2032017

Amran, A., Perkasa, M., Satriawan, M., Jasin, I., \& Irwansyah, M. (2019). Assessing students 21 st century attitude and environmental awareness: promoting education for sustainable development through science education. Journal of Physics: Conference Series, 1157(1), 022025. https://iopscience.iop.org/article/10.1088/1742-6596/1157/2/022025

Andrade, J. \& Gonzales, J. (2019). Relación entre actitudes pro-ambientales y conocimientos ecológicos en adolescentes con relación al entorno rural o 
urbano que habitan. Revista Kavilando, 11(1), 105-118. https://www.kavilando.org/revista/index.php/kavilando/article/view/287 Araya, A., Salas, S., \& Campos, C. (2019). Percepción Futura Del Humedal "El Culebrón", Coquimbo (Chile): Una Experiencia Educativa Interdisciplinaria. Revista Luna Azul, 49, 220-240. https://doi.org/10.17151/luaz.2019.49.13

Bamberg, S. (2003). How does environmental concern influence specific environmentally related behaviors? A new answer to an old question. Journal of environmental psychology, 23, pp. 21-32 https://doi.org/10.1016/S02724944(02)00078-6

Cerillo, A. (2020). La fauna recoloniza la ciudad ante el confinamiento por Corona

Virus. https://www.lavanguardia.com/natural/20200324/4874402309/animalesciudades-confinamiento-imagenes-curiosas.html

Castro, L., Bracho, S., Riatiga, H., Vera, E. \& Castro, D. (2016). Evaluación De La Problemática Ambiental en Poblaciones Marginales: Caso De Estudio Institución Educativa Del Departamento De Santander, Colombia. https://doi.org/10.16925/in.v12i19.1196

Çelik, A. (2020). Investigation of environmental awareness and cognitive structures of the 5th grade students. Marmara Cografya Dergisi, 41, 73-88. https://buscaintegrada.ufrj.br/EdsRecord/aph,141672654

Chuliá, E. (1995). La conciencia medioambiental de los españoles en los noventa. ASP Research Papers, 12, https://www.worldcat.org/title/conciencia-medioambiental-de-losespanoles-en-los-noventa/oclc/805655121

Corraliza, J. A., Bereguer, J., Moreno, M. y Martín, R. (2000). La investigación de la conciencia ambiental: Un enfoque sicosocial. Persona 
Sociedad y medio ambiente. https://www.juntadeandalucia.es/medioambiente/web/Bloques_Tematicos/P ublicaciones_Divulgacion_Y_Noticias/Documentos_Tecnicos/personas_soc iedad_y_ma/cap7.pdf

Dalhey, B. (2020). Daños colaterales de la COVID-19: el resurgir del plástico. The conversation. https://theconversation.com/danoscolaterales-de-la-covid-19-el-resurgir-del-plastico-137803

Danielraja, R. (2019). A Study of Environmental Awareness of Students at Higher Secondary Level. Shanlax International Journal of Education, 7(3), 6-10. https://files.eric.ed.gov/fulltext/EJ1245148.

Dourojeanni, M. (2020). Impactos ambientales de la pandemia en el Perú. Actualidad Ambiental. https://bit.ly/32lcvOz

Dutcher, D., Finley, J., Luloff, E. y Johnson, B. (/2007). Connectivity with nature as a measure of environmental values. Environment and behavior, 39, 2007, pp. 474-493. https://psycnet.apa.org/record/2007-11174-002

Educa (2021). Educación ambiental para niños y niñas: proteger el entorno y evitar otra pandemia [Mensaje en un blog] Recuperado de https://www.educo.org/Blog/Educacion-ambiental-para-ninos-protegepandemia

Espejel Rodríguez, A., \& Flores Hernández, A. (2017). Experiencias Exitosas De Educación Ambiental en Los Jóvenes Del Bachillerato De Tlaxcala, México. Revista Luna Azul, 44, 294-315. https://doi.org/10.17151/luaz.2017.44.18

García, G., Jordano, J., Lozano, B. y Nogueira, A. (2020). Observatorio de políticas ambientales 2020. Editorial CIEMAT. 
http://www.pucv.cl/uuaa/site/docs/20200728/20200728175638/2020_opam. pdf

Gaizkale, A. (2017). Programa Piloto De Reciclaje en Colegios Del Estado Anzoátegui, Venezuela. Saber: Revista Multidisciplinaria Del Consejo de Investigacion, 29, 523-533.

Gomera, A., Villamandos, F., \& Vaquero, M. (2013). Construcción De Indicadores De Creencias Ambientales a Partir De La Escala Nep. Acción Psicológica, 10(1), 149-160. https://doi.org/10.5944/ap.10.1.7041

Izquierdo, L., Ramón, E., Ruíz, R., \& Díaz G. (2010). Programa de educación ambiental, prevención y mitigación de riesgos por inundaciones aplicado en el colegio de Bachilleres de Tabasco, plantel no 28. Revista de La Alta Tecnología y Sociedad, 4(1), 1-12.

Kilbourne, E. y Polonsky, J. (2005). Environmental attitudes and their relation to the dominant social paradigm among university students in New Zeland and Australia. Australasian marketing journal, 13(2), 2005, pp. 3748. http://dro.deakin.edu.au/view/DU:30016262

Koyunlu, Z. (2020). Analysis of Short Films of Prospective Teachers on Environmental Awareness. International Electronic Journal of Environmental Education, 10(2), 136-146. https://eric.ed.gov/?id=EJ1256136

Kurniawan, E. \& Sari, N. (2019). Development Strategy of Cadres Students on School Based Environmental and Disaster Awareness. IOP Conference Series: Earth \& Environmental Science, 243(1), 012082. https://iopscience.iop.org/article/10.1088/1755-1315/243/1/012082

Laso, S., Ruiz, M. \& Marbán, M. (2019). Impacto de un programa de intervención metacognitivo sobre la Conciencia Ambiental de docentes de Primaria en formación inicial. Revista Eureka Sobre Enseñanza y 
Divulgación de Las Ciencias, 16(2), 1-20. https://doi.org/10.25267/Rev_Eureka_ensen_divulg_cienc.2019.v16.i2.2501 Leite, A., \& Lopes, R. (2020). Ressignificando O Ensino De Geografia Através De Práticas De Solo. Geografia,45(1), 117-139. https://dialnet.unirioja.es/servlet/articulo?codigo $=7583828$

Maloney, M. P. y Ward, M. P. (1973). Ecology: let's hear from the people. An objective scale for the measurement of ecological attitudes and knowledge. American psychologist, 30, 1973, pp. 787-790. DOI:10.1037/H0034936

Martin, A. R., Gheorghescu, I. C., Sandu, A., \& Salasan, C. (2019). Teaching Air Pollution to Students Aged 6-14: A Methodological Approach. Agricultural Management / Lucrari Stiintifice Seria I, Management Agricol, 21(3), 417-421. https://www.cabdirect.org/cabdirect/abstract/20203188421

Martínez García, R., Caballo Villar, M. B., \& Varela Crespo, L. (2020). El ocio en el medio natural como promotor de la conexión emocional con la naturaleza. Un estudio en clave ambiental con adolescentes pontevedreses (Galicia-España). Pensamiento $\quad$ Educativo, 57(2), 1-16. https://doi.org/10.7764/PEL.57.2.2020.6

Matthies, E. y Blöbaum, A. (2007). Impact of environmental concern and ecological norms. en T. Gärling y L. Steg (eds.). Threats from car traffic to the quality of urban life, Elsevier, Amsterdam, 2007, pp. 251-271.

Minedu (2020). Plan Nacional de Educación Ambiental 2016-2021 PLANEA. http://www.minedu.gob.pe/educacionambiental/ambiental/sumilla_planea.php

Ministerio del Ambiente (2021). Ambiente en Acción. Portal de transparencia. https://www.minam.gob.pe/ambienteenaccion/ 
Ministerio del Ambiente (2021). Programa GLOBE Perú - ConCiencia Ambiental desde la Escuela. Portal de Transparencia. https://www.minam.gob.pe/educacion/voluntariado/programa-globe-peruconciencia-ambiental-desde-la-escuela/ Miranda, D. (2017). Medición de la conciencia ambiental en estudiantes de ciencias agrarias de la Universidad Nacional de Tumbes. Revista de Humanidades \& Inovação $\quad$ v. 4 n. 2 (2017). ISSN: 2358-8322 https://revista.unitins.br/index.php/humanidadeseinovacao/article/view/303 Narain, U. (2020). Contaminación atmosférica: confinada pero no detenida por la COVID $19 . \quad$ Banco Mundial. https://www.bancomundial.org/es/news/immersive-story/2020/07/01/airpollution-locked-down-by-covid-19-but-not-arrested Nunes, F., Cruz A. \& Pastorio, E. (2020). Gestão dos resíduos sólidos em Escola do Campo: experiência de educação ambiental na EMCEF de São Gabriel/RS. Revista Monografias Ambientais, 19, 1-16. https://doi.org/10.5902/2236130843415

ONU-PNUMA (2020). Trabajar con el medio ambiente para proteger a las personas. Covid ttps://wedocs.unep.org/bitstream/handle/20.500.11822/32218/UNEPCOVID _SP.pdf?sequence $=15$

Organización Panamericana de Salud (2021). Determinantes Ambientales de Salud. https://www.paho.org/es/temas/determinantes-ambientales-salud Ohtomo, S. y Hirose, Y. (2007). The dual-process of reactive and intentional decision making involved in eco-friendly behavior. Journal of environmental psychology, 27 (2), 2007, pp. 117-125. Doi: 10.1016/j. jjenvp.2007-01.005.

Punzalan, H., Signo, C., Signo, C. \& Marasigan, C. (2019). Environmental Awareness of Selected Urban and Rural High School Students in the 
Philippines. Journal on School Educational Technology, 15(2), 15-25. https://eric.ed.gov/?id=EJ1239347

Ranniko, P. (1996). Local environmental conflicts and the change in environmental consciousness. Acta sociologica, 39(1), 1996, pp. 57-72. https://www.jstor.org/stable/4194805

Robina, R., \& Medina, A. (2019). Transforming students' environmental attitudes in schools through external communities. Journal of Cleaner Production, 232(1), 629-638. DOI: 10.1016/j.jclepro.2019.05.391

Rogayan, V. \& Nebrida, D. (2019). Environmental Awareness and Practices of Science Students: Input for Ecological Management Plan. International Electronic Journal of Environmental Education,9(2), 106-119. https://www.researchgate.net/publication/333667252_Environmental_Awar eness_and_Practices_of_Science_Students_Input_for_Ecological_Managem ent_Plan

Ruiz, J. (2006). Mentalidades medioambientales: los discursos sobre el medio ambiente de los andaluces residentes en zonas urbanas. Papers, 81, 2006, pp. 63-88. https://raco.cat/index.php/Papers/article/view/55650

Santacruz, A. (2018). La estrategia del debate en el fortalecimiento de la conciencia ambiental. Revista científica Investigación Valdizana Vol. 12 Núm. 4 (2018). DOI: https://doi.org/10.33554/riv.12.4.153

Santos, E., Dias, P. \& Afonso, T. (2014). Consciência Ambiental, Atitudes E Intenção De Uso Das Sacolas Plásticas Não-Recicláveis. Revista de Gestão Ambiental e Sustentabilidade (GeAS), 3(1), 71-87. https://doi.org/10.5585/geas.v3i1.112

Santos, E. \& Passarella, C. (2013). Ensinando E Aprendendo Educação Ambiental Com Jovens. Revista Ciência Em Extensão,9(3), 82-93. https://core.ac.uk/download/pdf/233140334.pdf 
Schultz, W. (2002). Inclusion with nature: the psychology of human-nature relations. En P. Scmuck y P. W. Schultz (eds.). Psychology of sustainable development, Norwell, Kluwer Academic, 2002. DOI:10.1007/978-1-46150995-0_4

Süklüm, N. (2019). Yeşil Muhasebe Farkındalığının Sosyal Sorumluluk Çerçevesinde Analizi: Çanakkale Onsekiz Mart Üniversitesi Örneği. Muhasebe ve Vergi Uygulamalari Dergisi (MUVU) / Journal of Accounting \& Taxation Studies (JATS), 95-112. https://doi.org/10.25095/mufad.625745

Tonello, G. \& Valladares, N. (2015). Conciencia ambiental y conducta sustentable relacionada con el uso de energía para iluminación. Gestión y Ambiente, 18(1), 45-59. https://core.ac.uk/download/pdf/80374287.pdf Torres, V. (2018). Nivel de educación ambiental de los estudiantes de secundaria de la Amazonía del Perú. Pacarina del Sur, año 10, núm. 37, $\begin{array}{llll}\text { octubre-diciembre, } & 2018 . & \text { ISSN: }\end{array}$ www.pacarinadelsur.com/index.php?option=com_content $\&$ view=article\&id $=1679 \&$ catid $=10$

Van Liere, y Dunlap, E. (1981). Environmental concern: does it make a difference how it's measured? Environment and behavior, 13(6), 1981, pp. 651-676.

https://www.researchgate.net/publication/232497667_Environmental_Conc ern_Does_it_Make_a_Difference_How_it's_Measured

Widiyawati, Y. (2020). Global warming \& climate change: integration of socio-scientific issues to enhance scientific literacy. Journal of Physics: Conference Series, 1511(1), $\quad 012071 . \quad$ DOI: $\underline{10.1088 / 1742-}$ $\underline{6596 / 1511 / 1 / 012071}$

Yeşilyurt, M., Ozdemir Balakoğlu, M., \& Erol, M. (2020). The Impact of Environmental Education Activities on Primary School Students' 
Environmental Awareness and Visual Expressions. Qualitative Research in Education (2014-6418), 9(2), $188-216$. https://doi.org/10.17583/qre.2020.5115

Yilmaz, Y. (2019). An Examination of the Relationship between Primary School Students' Environmental Awareness and Basic Science Process Skills. Educational Research and Reviews, 14(4), 140-151. https://doi.org/10.5897/ERR2018.3663 